\title{
Generating Functional Mockup Units from Software Specifications
}

\author{
Uwe Pohlmann, \\ Wilhelm Schäfer \\ Software Engineering Group, \\ Heinz Nixdorf Institute, \\ University of Paderborn, Germany \\ [upohl|wilhelm] @upb.de
}

\author{
Hendrik Reddehase, Jens Röckemann, \\ Robert Wagner \\ Solunar GmbH, \\ Gütersloh, Germany \\ [reddehase | roeckemann] @solunar.de \\ wagner@solunar.de
}

\begin{abstract}
This paper presents an approach to use the Functional Mockup Interface (FMI) for integration of classical controller specifications and statechartbased specifications of real-time critical message exchange protocols. The Functional Mockup Unit (FMU) is automatically generated from the specification. Using the generated FMU we are able to exploit simulation facilities as provided by Modelica/Dymola.

Keywords: Systems Engineering, Software Engineering, MechatronicUML, FMI, FMU, Modelica
\end{abstract}

\section{Introduction}

In today's globalized world market forces demand products to provide for more and more unique features. In so-called mechatronic or embedded systems these features are often realized (mainly) by software. For example, many new features which were recently introduced in the automotive industry are largely software driven.

In addition, very advanced new features will depend on extensive communication between currently still independently operating individual components. For example, intelligent lighting systems in cars will combine information about the environment obtained from their own sensors with those collected by other cars to save energy but also to avoid glaring other drivers. Similar examples exist for transportation systems in general but also for household appliances or in the production industry [25]. Here, possible significant energy savings are one main motivation to introduce so-called smart grids.

The resulting high amount of software enabling communication between a large number of components combined with the software controlling individual components makes those systems more complex than today. This requires significant changes in the way software is developed today. This is especially true as the software controlling individual components is usually dealing with continuous variable values and developed by control engineers whereas software controlling communication is handling discrete input and output signals often using asynchronous communication and is developed by software engineers. In addition, electrical and mechanical engineers bring in expertise about the underlying hardware system constraints which have to be considered when developing the software.

As these systems are usually deployed in safetycritical environments, high quality of the software is an absolute must [21]. However, in the past, an overall validation of systems under construction was not possible until implementations had been finished, i.e., after all hardware and software parts had been built and integrated into the final product. The above mentioned different disciplines use their own models and formalisms to describe the corresponding parts of the system under development, e.g. feedback controllers are described using differential equations and communication protocols are described using statecharts. This development process hinders early (formal) verification and simulation of system models to detect errors in the design phase as early as possible and to avoid costly error removal in later development stages.

In this paper we focus on supporting simulation based on model-driven development especially considering cross-discipline development between control and software engineering. In contrast to other approaches like $[22,8,26]$, we use 
a discrete system model which enables the detailed specification of timing issues when specifying communication protocols, because message transfer specified by those protocols is real-time critical. Proper functioning of the system does not only depend on the correct order of messages sent and received but also on their timely delivery.

This paper presents how we employed the Functional Mockup Interface (FMI) and the Functional Mockup Units (FMU) in order to integrate discrete model-based real time protocol specification with controller design and appropriated simulation facilities using Modelica/Dymola.

The approach has been developed as part of the ENTIME project (ENTIME is the German acronym for 'Design Methods for Intelligent Mechatronics'). The project aims at the development of a seamless methodology reaching from conceptual design to concrete implementation of mechatronic systems. It is carried out in close cooperation with nine industrial partners. To support simulation of the physical models and corresponding feedback loops together with specifications of real-time protocols, the main challenge was to provide the needed tool support, because the project collaborators use different modeling and simulation tools in their industrial practice.

The paper is organized as follows. In the next section we illustrate the use of MechatronicUML, a domain specific modeling language enabling protocol specifications including sophisticated realtime constraints. The example which we use in the paper, is a miniature robot called BeBot which is a small mechatronic systems with a focus on ad-hoc communication. In Section 3, we give a brief and informal introduction to the concepts of the FMI standard, sketch our implementation of MechatronicUML according to the FMI standard for model exchange by means of the example, and present our tool support. Section 4 discusses related work in more detail. The paper closes with a conclusion and an outlook on future work.

\section{$2 \quad$ Specification of Protocols}

The specification language which we use is called MechatronicUML [3]. It has been developed by a large joint project between engineers and people from computer science. The project is the collaborative research center self-optimizing systems in mechanical engineering which is funded by the German national science foundation since 2002 (http://www.sfb614.de/en/).

\section{$2.1 \quad$ Running Example}

The example is the scenario of a so-called obstacle avoidance maneuver which is performed by a BeBot. BeBot [11] is a sophisticated intelligent miniature robot, developed by the Heinz-Nixdorf Institute. Figure 1 shows a picture of a BeBot. In our scenario, the BeBot uses three sensors which detect obstacles in front, left and right of its current position. Further, the BeBot has a gyroscope which measures its current angle position with respect to the outside world. Three components of the BeBot are active when it performs obstacle avoidance. These components are (1) an exploration component which starts or stops the exploration of the environment, (2) a navigation component which steers the BeBot around an obstacle based on the given sensor inputs and (3) an obstacle detection component which receives the input from the three sensors and transforms them into corresponding messages which are received by the navigation component.

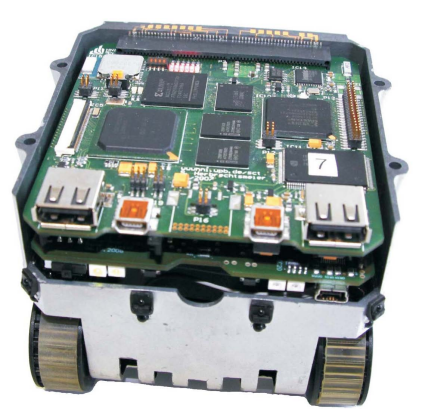

Figure 1: BeBot Robot [11]

As a consequence, the decision if and how an obstacle avoidance maneuver has to be performed depends on (extensive) asynchronous communication between these three components. For example, the navigator which knows the actual angle to the outside world, informs the obstacle detection component which sensor values are relevant. The obstacle detection component must not send messages when a turn is performed, because sensor values are not correct when the BeBot spins.

Figure 2 illustrates how a BeBot will find its way out of the shown maze. 


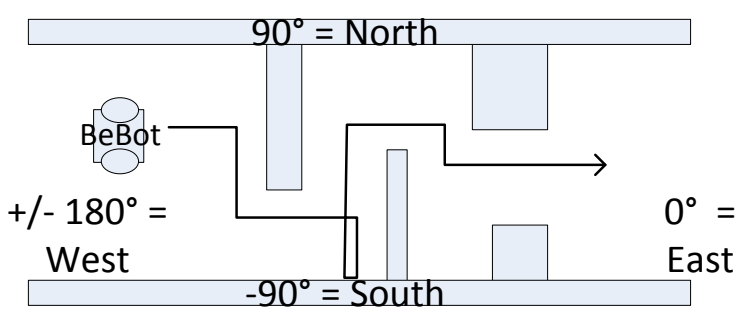

Figure 2: BeBot Obstacle Avoidance Maneuver

\section{$2.2 \quad$ Structure Model}

In MechatronicUML the system model is structured hierarchically and consists of either atomic components or of structured components. Atomic components implement their behavior directly and structured components are a composition of other components. The component model of MechatronicUML differs from other componentbased approaches, like [27], as MechatronicUML employs active components, i.e. the behaviour of each component is specified by a real-time statechart (see below) and executed by a single thread [3].

Each component has interaction points, called ports for accessing their functionality. Discrete ports, shown as rectangles, are used for sending and receiving asynchronous messages. Each message is typed over a message type. Further, discrete message ports have the causality in $\nabla$, out $\square$, or in/out $\square$. Discrete in-ports can only receive messages, discrete out-ports can only send messages and in-out-ports can receive and send messages. A continuous port, shown as a triangle is either a continuous in-port $\varangle$, or a continuous out-port $\triangleright$. It sends or receives signal values which are typed as Boolean, Int, or Real.

Figure 3 shows the internal structure of the BeBot_SW component. It consists of three atomic components. The component Exploration is responsible for starting and stopping the exploration scenario. It is connected via its port sender to the component Navigation. The Navigation component is responsible for actuating the BeBot. It can set the linear_speed and the angular_speed of the BeBot. The Navigation component is connected to the ObstacleDetection component via its discrete port master. The ObstacleDetection component transfers the continuous signal values of the sensors front, left, and right to asynchronous messages. These messages inform the Navigation component if it has to perform an obstacle avoidance maneuver.

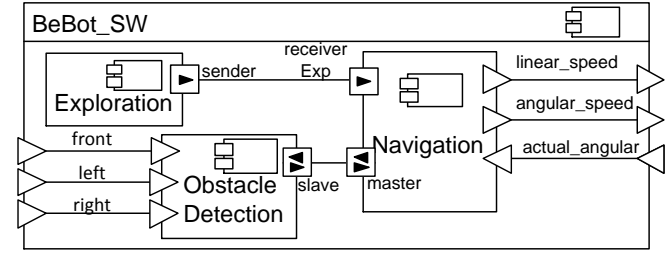

Figure 3: Component Type of the BeBot Software

\subsection{Real-Time Properties}

Real-Time properties are specified by clocks. In MechatronicUML a clock is a first-class realvalued entity and is used to synchronously measure the duration of time during execution. It can be reset to zero, which is marked by the keyword reset, with any state- or transition-action. At the beginning of the simulation clocks start with a zero-value. In contrast to delayed transitions of State Graph2 [22], or the after, before-construct of Stateflow [23], or the relative time event after of UML, a clock is not automatically reset when the system state changes. At any point in time, a clock can be read. The value of the clock represents the continuous-time since the last reset [2]. This semantics simplifies the specification of more complex real-time behavior and constraints. It is possible to compare clock values with time constants. We use clocks to specify transition guards, transition deadlines and time invariants of states.

\subsection{Discrete Behavior Model}

MechatronicUML uses Real-Time Statecharts to specify protocols of message exchange between different components, i.e. the order of message invocation and its corresponding time constraints. Besides elements from UML state machine formalism Real-Time Statecharts use syntactic elements like clocks and corresponding clock constraints as extended transition guards as defined by timed automata. In MechatronicUML each discrete port has its own statechart. The behavior of a component is given by the parallel composition of all statecharts of all its ports. In addition, it is possible to add synchronization channels like in timed automata to synchronize the behavior of the different port statecharts.

Time-invariants from timed automata constrain when and how long a statechart is allowed to stay in a particular state. We define the maximum time for evaluating and executing a transitions by a deadline. We use clocks as guards of 
transitions, deadlines of transitions, and time invariants of states. The operational semantics of Real-Time Statecharts is formally defined by [12] and is based on timed automata.

It enables the application of formal verification techniques like real-time model checking [14] with tools like UPPAAL [4]. For instance we specify in our example in Figure 4 the safety property that each turn maneuver may not last longer than 5 seconds. Therefore we use the time invariant $c 0<5$.

Figure 4 shows the Real-Time Statechart of the Navigation component. It consists of the parallel composition of the port statecharts receiverExp and master. The statechart in region receiverExp describes how the received messages from component Exploration are processed. At the beginning the statechart is in its initial state Stop and the parallel statechart master is in the state Halt. When the upper statechart gets the asynchronous message start the outgoing transition fires, if the synchronization channel go is available. The synchronization channel go is available if the sender transition, marked by the "!", and the receiving transition, marked by the "?" can fire. If both transition can fire both transitions fire together in an atomic way. This means either both fire or none of them. Because there are no more conditions on the transitions they fire and the statechart gets in the states Start and Go.

When the statechart master enters the state Go the output signal linear_speed of the BeBot is set to the value 0.1 and the angular_speed is set to 0 . In the state Go the BeBot drives forward until the ObstacleDetection sends the message obstacleFront. In this case the BeBot turns right to a southward direction and drives forward until the left sensor signals that there is no more obstacle at the left side. If there is no more obstacle, the BeBot turns back in an eastward direction and drives forward until the next obstacle occurs in front of it. If there is an obstacle at the left side until the BeBot reaches the corridor boarder, the BeBot performs a U-turn and drives forward until the right sensor signals that there is no more obstacle at the right side. These steps are carried out in a loop until the Exploration component sends the stop message.

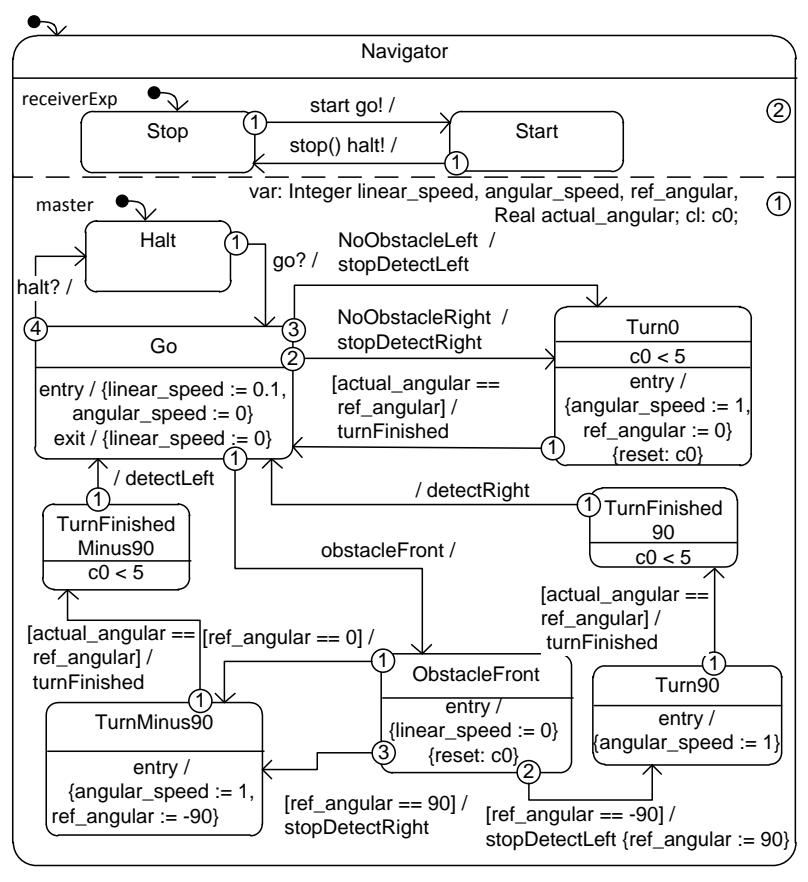

Figure 4: Real-Time Statechart the Navigator

\subsection{Asynchronous Communication}

The shown Real-Time Statechart formally defines the protocol definition of the message exchange and the corresponding timing constraints. Messages are sent when a transition fires. Messages which should be sent are shown behind the slash (/) and messages which should be consumed are shown before the slash. The connector may have a delay or a message could be lost. For the sake of simplicity of the figure above and due to lack of space, we omit the specification of the connector here. The receiver port of a message stores a received message in a mailbox. This is implemented as a queue and has a fixed size which is defined by the modeler during design time. Each message type has its own mailbox. Thus, the receiver can test directly if a needed message is available without searching the whole queue. Each message type could have an arbitrary number of parameters, which are packaged in the message when a transition fires. The receiver transition can read and process the parameters when it fires and consumes the message. Messages remain in the mailbox until a transition consumes and destroys it.

\subsection{Further Features of MechatronicUML}

As explained, MechatronicUML [3] mainly focuses on the discrete parts of systems. The language 
especially addresses the specification of complex communication protocols with hard real-time requirements [9].

The structure of a mechatronic system is defined by a component-based development approach. It is possible to distinguish between discrete software components and continuous software components like controllers. MechatronicUML has clear interfaces between discrete system parts and continuous system parts.

The behavior of continuous components including their communication protocols is specified by an extension of our Real-Time Statecharts in the sense of hybrid automata. However, in contrast to hybrid automaton approaches [1, 19] we abstract from detailed definitions of controllers.

This abstraction together with some constraints on the parallel composition of port statecharts enables formal verification of the behavioral specification using model checking. We employ the model checker UPPAAL to verify safety properties like deadlock freeness, state reachability or end-to-end response time. MechatronicUML models can be verified automatically. We also prove by model checking that a mailbox will not overflow (see above). However, formal verification is beyond the scope of this paper and we refer to $[15,13]$ for further details.

\section{Generating FMUs Software Specification}

This section shows how to generate an FMU.

\subsection{FMI/FMU Fundamentals}

Using different tools when designing the models leads to compatibility problems when you want to simulate all models in combination. To address this problem, the ITEA2 project MODELISAR has defined the FMI as an open standard for model exchange and co-simulation between multiple software systems. The FMI is used to create an instance of a model which can be loaded into any simulator providing an import function for FMI [7]. The FMI for Co-Simulation allows to couple several simulation tools [6].

A software instance compatible to the FMI is called an FMU. An FMU is basically a zip-archive with a "*.fmu" file extension. The information required for the simulation environment is collected in an XML-file called modelDescription.xml. In addition, this file also includes a list of all variables available for data exchange between the simulator and the FMU. Furthermore, the standard defines functions that are used for the interaction between a model and the simulator. To provide an FMU, the FMU provider has to implement these functions using the $\mathrm{C}$ language.

\subsection{Generating C-Code from MechatronicUML}

This section sketches the C-code generation techniques for MechatronicUML models. The generated code may be used for a concrete microcontroller target platform or - as this paper shows for an FMU implementation.

\subsubsection{Generating C-code from the Structure Model}

For each atomic component of the MechatronicUML model, we generate a header file and a corresponding implementation file. A component is mapped to a structure containing pointers to nested sub-components, variables, and clocks required for the associated statechart. In addition, corresponding code for the ports is generated. The discrete port implementation is used for inter-component communication. For this purpose, a discrete port implements an array of message queues. A queue stores messages of one specific type. For parametrized message types additional structures are generated in order to encapsulate the parameter values. For continuous in-ports we generated a variable with the causality input and for continuous out-ports we generate a variable with the causality output. The continuous port type is mapped to a corresponding FMI data type, e.g. Boolean to fmiBoolean. Via the input and output variables the FMU can be connected to other FMUs or Modelica/ Simulink components.

Our code is intended to run also on small 8-bit processors with only a few kilobytes of memory. This is too little to support both a real-time operating system and the control software. Hence, the control software is executed standalone on the processor and to support multiple communicating components on one processor, the components are processed in a cycle using a simple task loop implementation. Note that for future work we will intro- 
duce a real-time operating system with more sophisticated task management and scheduling features for larger systems with 16 - and 32-bit processors.

Listing 1 shows the execution sequence of our example. The information about a component is passed as an argument, allowing for multiple components of the same type to exist in one environment. In every processing cycle, a component statechart may exchange messages with other components by sending and receiving them. After every component has been processed, a synchronization step is performed where raised events are delivered to the target components.

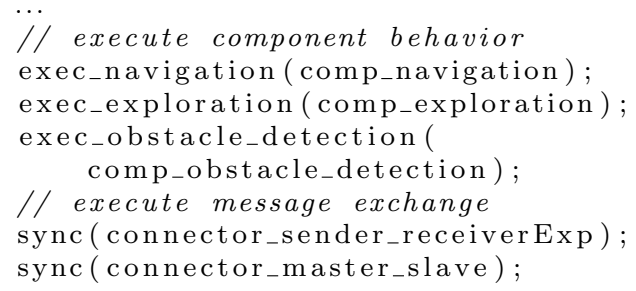

Listing 1: BeBot Execution Sequence

\subsubsection{Generating C-Code from the Discrete Behavior Model}

There are several implementation techniques for statecharts, but in most cases all the techniques are variants and combinations of (1) the state table, (2) the object-oriented state design pattern, and (3) the simple switch-case statement implementations. (1) The state table implementation maps directly to a state table representation in the code. As it is not hierarchical, it needs extensions for nested states and parallel regions and requires a large state table representation with a complicated initialization. Hence, the code is less readable. (2) The state design pattern simplifies the implementation of statecharts. However, it has also to be extended for hierarchical statechart implementations. In addition, the implementation is straightforward in $\mathrm{C}++$, but it is rather complex in $\mathrm{C}$, because of the needed mapping for inheritance and polymorphism. Therefore, we decided to generate nested switch-case statements (3). The implementation technique of switch-case statements is quite simple, it can be easily coded in $\mathrm{C}$, and it has a small memory footprint since only one state variable is necessary to store the current state of a state machine. Furthermore, nested switch-case statements allow us to implement hierarchical statecharts in a quite intuitive and readable way, which ensures traceability between the model and the generated code.

Listing 2 shows a code excerpt from the generated program for the BeBot example shown in Figure 4. It gives an impression of the generated C-code for a transition from state ObstacleFront to state TurnMinus90.

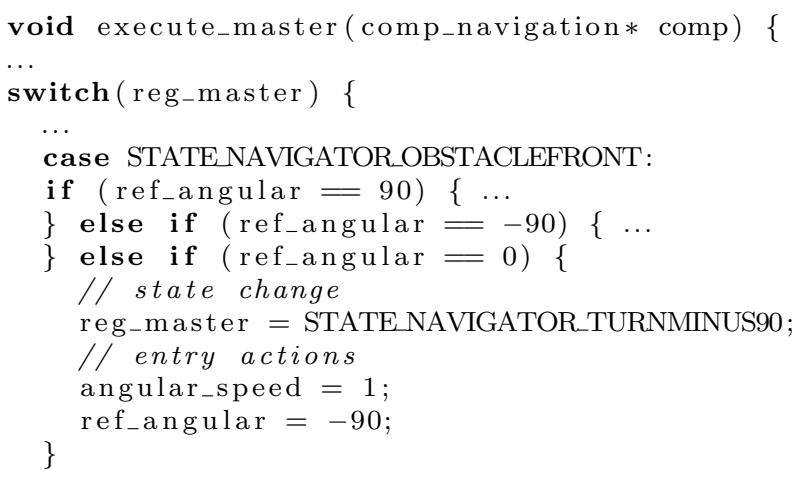

\section{Listing 2: Excerpt from navigation.c}

For each region of a statechart, we declare an Integer variable to keep the current state of this region. Within each case-statement, a sequence of mutually exclusive if-statements is used to determine whether one of the state's outgoing transitions can fire. In order to enable a transition, the existence of events and Boolean expressions generated from conditional guards, clock guards, and synchronization channels have to be evaluated. As transitions have priorities in MechatronicUML to prevent non-deterministic behavior, the generator sorts the transitions according to their priorities before generating the appropriate code. If a transition is enabled, it fires, i.e. the new state is set and the appropriate exit and entry actions are executed. A state may also contain do-actions and inner regions which are executed if no transition is enabled. For this case, a final else-block is created. Note, the presented program is executed once in each cycle. In order to use the generated $\mathrm{C}$-code for an FMU implementation, we have to implement the required interfaces from the FMI standard.

\section{$3.3 \quad$ FMI/FMU Wrap Up}

We employ the FMU SDK from QTronic [16]. Figure 5 shows the relations and dependencies between the FMI standard, the QTronic FMU SDK, and our statechart implementation. 


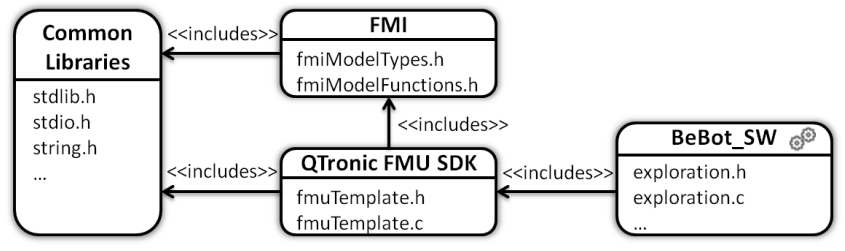

Figure 5: Implementation Dependencies

The basic implementation of the FMI is provided by the FMU SDK. To implement MechatronicUML, our code generator creates a header file and an implementation file for each component taking the FMU SDK into account.

The FMU SDK implements the FMI standard by delegating some of the tasks to supplementary functions that have to be implemented by the user. In our case, these implementations are also generated automatically by our code generator. The most important function is eventUpdate, as it is used to execute the statecharts. Since the eventUpdate-function is called by the FMIfunction fmiEventUpdate whenever an event occurs during a simulation, we are able to react on changes in the simulation model. We use Time Events from the FMI to control the execution of a statechart at a regular interval and map each clock to an fmiReal variable. Since the current simulation time is passed to the FMU as a parameter, the current simulation time is assigned to the clock variable to reset a clock. Evaluating is done by calculating the elapsed time since the last reset. The difference between the current simulation time and the affected clock variable is used to evaluate clock constraints, deadlines and invariants upon appropriate actions are taken.

In the FMI standard, direct access to the data stored within the model is not possible, even if the source code is provided. Instead, a reference number is associated with each variable in the description file. Therefore, the FMU SDK stores variables of the model in four arrays of the types fmiReal, fmiInteger, fmiBoolean, and fmiString and references them by using indices. This is an efficient implementation of the FMI standard, but it is not useful for target-specific code that does not serve as an FMU implementation. Further, it is not easy to read and to understand the code. Therefore, we generate placeholders for the variables of our model. For the FMU implementation we generate preprocessor macros, which map the placeholders to FMU SDK compliant array access statements.

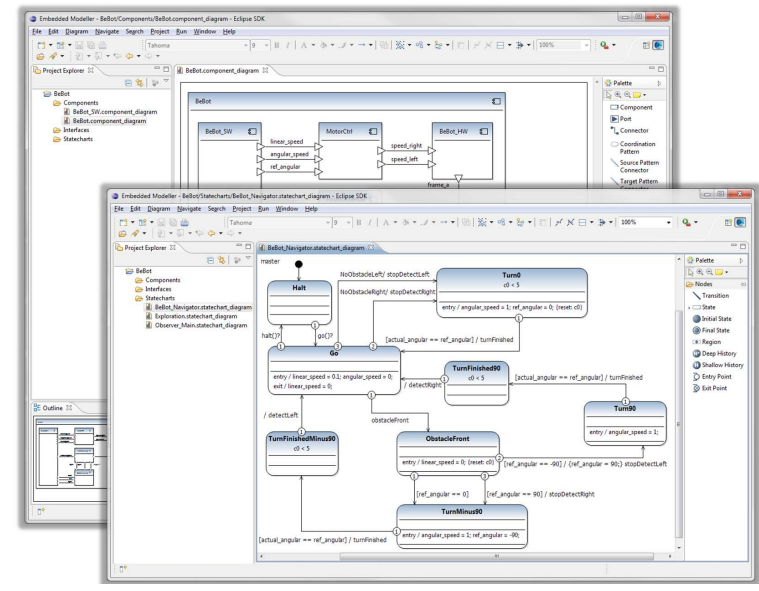

Figure 6: EMBEDDEDMODELLER

In case of other targets, e.g. microcontrollers, we generate preprocessor macros mapping the placeholder to more suitable structures and variables.

\subsection{Tool Support}

We provide our tool support in form of an Eclipse modeling tool suite which is called EMBEDDEDModeller. The EMBEDdEdMOdELleR provides several diagram editors and supports software specifications based on MechatronicUML as explained in the previous sections. Figure 6 shows the editors for Real-Time Statecharts and structured component diagrams.

For generating C-code and the corresponding FMU description file, we used a template-based code generator framework. To create the FMU with all resources, the batch script provided with the FMU SDK is executed. Firstly, the batch file creates a temporary directory with the desired directory structure for the FMU under construction. Secondly, it compiles the sources and copies all needed files to the corresponding folders. Lastly, the batch packages the processed directory and saves it within the ${ }^{*}$.fmu file.

To simulate the designed BeBot software, we generated the FMU for our software specification BeBot_SW and created the Modelica model of the mechanical and control engineering parts of the BeBot within Dymola. The FMU was imported and connected to the hardware model of the BeBot. Since the continuous ports of the MechatronicUML serve as an interface to continuous components in general, we are able to connect our FMU, i.e. our discrete software component, to the provided BeBot feedback controllers. We simu- 
lated the integrated model in Dymola successfully. Note, our approach is not limited to Modelica / Dymola as the FMI standard is tool-independent. Therefore, it is possible to simulate software specifications with any other simulation tool which supports the FMI model exchange standard.

\section{Related Work}

This section presents related work. We focus on approaches which can be used to simulate hybrid systems and where the discrete behavior is state-based. Further, we currently use the FMI for model exchange and not for co-simulation. Therefore, we do not discuss other approaches for co-simulation or distributed simulation like CODIS [5], TISC [20] or FMI co-simulation [6].

\subsection{Statecharts in Modelica}

Currently, state-based behavior can be modeled in Modelica with the library State Graph2 or algorithmic code is generated from SimulationX or ModelicaML.

State Graph2 is a Modelica library [22] which provides classes for states (Step), hierarchical and parallel behavior (Parallel), and transitions (Transition). With these elements it is possible to model complex behavior like Harel's wrist watch example. In contrast to MechatronicUML, StateGraph2 has no concepts for clocks, clock constraints, time invariants, and deadlines to specify and constrain timing behavior. A modeler could manually implement such behavior in Modelica. Further, State Graph2 has no concept for asynchronous message-based communication. We are currently working on such a library extension. However, as State Graph2 is modeled with equations and these equations are sorted before the model is simulated, the modeler can hardly influence the resulting C-code generated by Dymola. So, it is difficult to compare this code with real target source code. The FMI C-code is the same as the target source code except for the interface definition .

SimulationX has its own state-based language which follows the ideas of UML state machines and supports a subset [8]. In contrast to RealTime Statecharts, SimulationX does not support parallel behavior, timing behavior, and coordination of distributed components by asynchronous communication. Timing behavior is supported in a limited way, as transition firing could be constrained to a time interval from the moment when the source state of the transition is entered. In MechatronicUML we use, like timed automata, clocks, clock constraints, time invariants, and deadlines to specify and constrain the timing behavior of our models. Messages are only available within a statechart in SimulationX. They do not support an arbitrary number of parameters, and messages are lost when a transition cannot react on the event immediately. Therefore, it is difficult to specify coordination of distributed components. SimulationX generates Modelica algorithm code from its state machines.

ModelicaML is a UML Profile [26] which enables to use UML Classes and Properties to specify Modelica models. State-based behavior is modeled by UML state machines. The code generation mechanism supports nearly all UML state machine constructs [24]. The Modelica code is generated like SimulationX to the Modelica algorithm section. As UML has no concept for clocks, clock constraints, time invariants, and deadlines, ModelicaML does not support them either. Asynchronous messages between components can be simulated via an external C-function [24]. As ModelicaML has all freedoms of Modelica, it is not possible to verify the resulting models efficiently.

\subsection{MATLAB Simulink/Stateflow}

MATLAB has an own state-based modeling language called Stateflow, which can be combined with its simulation platform Simulink. Stateflow supports many features from UML state machines and can be combined with the whole capabilities of the MATLAB platform via its action language. It is only possible to define formal semantics for restricted Stateflow models [17]. Stateflow does not provide first class modeling entities for specifying timing behavior, except simple after and before statements. Stateflow does not provide a concept of buffering messages. It is possible to model such elements with a combination of Simulink and Stateflow blocks, but this is complex, error prone, and hard to maintain manually [18]. It is possible to load FMU using the separate FMI toolbox of Modelon [10]. 


\section{Conclusion and Outlook}

This paper shows how it is possible to generate FMUs from a formal software specification language for cyber-physical system. As a result it is possible to perform software-in-the-loop tests by numerical simulation of hybrid systems. We describe the following problem that arises when providing a methodology and tool support reaching from conceptual design to concrete implementation of cyber-physical systems: The approach should support the overall system simulation for different industrial partners in a heterogeneous design tool environment. The partners provide simulation models for mechanical and control engineering parts of the system, but software simulation models are missing. The transformation of software specification to FMUs solves this problem.

As the main contribution, we describe how a software specification in MechatronicUML can be automatically translated to FMUs maintain the original MechatronicUML semantics and, thus, the verification results. In particular, we map the component-based structure, the asynchronous communication in form of Real-Time Statechart, and real-time properties in MechatronicUML to C-code, which is wrapped by the FMI. We implemented the generation of FMUs from a given MechatronicUML model using a modeldriven transformation approach. This combines the modeling and formal verification strengths of MechatronicUML with the advanced simulation capabilities of simulation tools like Dymola or Simulink. As a result of numerical errors we cannot guarantee that in different FMI import tools the different simulation runs have the same behavior. Therefore, the formal verification is important because it proofs every possible simulation run and guarantees that all paths are conform to the specification. It is up to further research to proof that our generation is correct and keeps the verified properties.

The shown transformation approach should be interesting for anyone who wants to test formal software specification by simulation against a model of the physical system. A transformation against the FMIs could be performed for other formal software specification languages like Petri nets for flow analysis or stochastic software models for testing performance or failure rates. Hereby, it would be possible to combine the strength of formal analysis and numeric simulation.
For future work, we plan to develop a concept to allow for communicating via messages between several FMUs. Further, we want to generate code against different hardware platforms to analyze the timing behavior. We want to integrate the behavior of an underlying middleware or real-time operating system into the simulation. We may use co-simulation for this purpose. The simulation of complex cyber-physical systems requires much computing time. We want to compare the performance of native Modelica simulations with integrated FMU simulations and try to enhance the performance of hybrid simulations. Currently, it is not easy to interpret the simulation results. Here a bisimulation concept would help. To show the result, a simulation run could be visualized in the statechart or the message exchange could be visualized by sequence diagrams.

\section{Acknowledgments}

This work was developed in the project 'ENTIME: Entwurfstechnik Intelligente Mechatronik' (Design Methods for Intelligent Mechatronic Systems). The project ENTIME is funded by the state of North RhineWestphalia (NRW), Germany and the EUROPEAN UNION, European Regional Development Fund, 'Investing in your future'.

\section{References}

[1] R. Alur, C. Courcoubetis, N. Halbwachs, T.A. Henzinger, P.H. Ho, X. Nicollin, A. Olivero, J. Sifakis, and S. Yovine. The algorithmic analysis of hybrid systems. Theoretical computer science, 138(1):3-34, 1995.

[2] R. Alur and D. L. Dill. A theory of timed automata. Theoretical Computer Science, 126:183235, 1994.

[3] S. Becker, C. Brenner, S. Dziwok, T. Gewering, C. Heinzemann, U. Pohlmann, C. Priesterjahn, W. Schäfer, J. Suck, O. Sudmann, and M. Tichy. The mechatronicuml method - process, syntax, and semantics. Technical Report tr-ri-12-318, Software Engineering Group, Heinz Nixdorf Institute University of Paderborn, 2012.

[4] G. Behrmann, A. David, and K. Larsen. A tutorial on uppaal. Formal methods for the design of realtime systems, pages 33-35, 2004.

[5] F. Bouchhima, G. Nicolescu, E. M. Aboulhamid, and M. Abid. Generic discrete-continuous simulation model for accurate validation in heterogeneous systems design. Microelectron. J., 38(67):805-815, June 2007. 
[6] MODELISAR Consortium. Functional mockup interface for co-simulation. version 1.0, 2010. www.functional-mockup-interface.org.

[7] MODELISAR Consortium. Functional mock-up interface for model exchange. version 1.0, 2010. www.functional-mockup-interface.org.

[8] U. Donath, J. Haufe, T. Blochwitz, and T. Neidhold. A new approach for modeling and verification of discrete control components within a Modelica environment. In Proceedings of the 6th Modelica Conference (Modelica 2008), Bielefeld, pages 269-276, 2008.

[9] S. Dziwok, C. Heinzemann, and M. Tichy. Realtime coordination patterns for advanced mechatronic systems. In Proceedings of the 14 th International Conference on Coordination Languages and Models (COORDINATION 2012), pages 166-180, June 2012.

[10] S. Gaaloul, B. Delinchant, F. Wurtz1, and F. Verdière. Software components for dynamic building simulation. In Proceedings of Building Simulation 2011: 12th Conference of International Building Performance Simulation Association, Sydney, Australia, pages 2278-2284, November 2011.

[11] J. Gausemeier, T. Schierbaum, R. Dumitrescu, S. Herbrechtsmeier, and A. Jungmann. Miniature robot bebot: Mechatronic test platform for self-x properties. In Proceedings of the 9th IEEE International Conference on Industrial Informatics (INDIN 2011), pages 451-456, July 2011.

[12] H. Giese and S. Burmester. Real-time statechart semantics. Technical Report tr-ri-03-239, Lehrstuhl für Softwaretechnik, University Paderborn, Paderborn, Germany, June 2003.

[13] H. Giese, S. Burmester, W. Schäfer, and O. Oberschelp. Modular design and verification of component-based mechatronic systems with online-reconfiguration. In Proceedings of 12th ACM SIGSOFT FSE, pages 179-188, 2004.

[14] H. Giese, M. Tichy, S. Burmester, W. Schäfer, and S. Flake. Towards the compositional verification of real-time uml designs. In Proc. of the 9th European software engineering conference held jointly with 11th ACM SIGSOFT international symposium on Foundations of software engineering (ESEC/FSE-11), 2003.

[15] H. Giese, M. Tichy, S. Burmester, W. Schäfer, and S. Flake. Towards the compositional verification of real-time uml designs. In Proceedings of 9th ESEC and 11th ACM SIGSOFT FSE, pages 3847. ACM Press, 2003.

[16] QTronic GmbH. FMU SDK 1.0.2, 2011. www.qtronic.de/de/fmusdk.html.
[17] G. Hamon and J. Rushby. An operational semantics for Stateflow. International Journal on Software Tools for Technology Transfer (STTT), 9(5):447-456, 2007.

[18] C. Heinzemann, U. Pohlmann, J. Rieke, W. Schäfer, O. Sudmann, and M. Tichy. Generating simulink and stateflow models from software specifications. In Proceedings of the International Design Conference (DESIGN 2012) Dubrovnik, Croatia, May 2012.

[19] T.A. Henzinger. The theory of hybrid automata. In Logic in Computer Science, 1996. LICS'96. Proceedings., Eleventh Annual IEEE Symposium on, pages 278-292. IEEE, 1996.

[20] R. Kossel, W. Tegethoff, M. Bodmann, and N. Lemke. Simulation of complex systems using modelica and tool coupling. In Proceedings of the 5th International Modelica Conference (Modelica 2006), pages 485-490, 2006.

[21] P. Marwedel. Embedded and cyber-physical systems in a nutshell. DAC. COM Knowledge Center Article, 2010.

[22] M. Otter, M. Malmheden, H. Elmqvist, S.E. Mattsson, C. Johnsson, D. Systèmes, and S.D. Lund. A new formalism for modeling of reactive and hybrid systems. In Proceedings of the 7th Modelica Conference (Modelica 2009), Como, Italy, pages 364-377, 2009.

[23] M. Pajic, Z. Jiang, I. Lee, O. Sokolsky, and R. Mangharam. From verification to implementation: A model translation tool and a pacemaker case study. In Proceedings of the 18th IEEE RealTime and Embedded Technology and Applications Symposium (RTAS 2012), Beijing, China, April 2012.

[24] U. Pohlmann and M. Tichy. Modelica code generation from modelicaml state machines extended by asynchronous communication. In Proceedings of the 4 th International Workshop on EquationBased Object-Oriented Modeling Languages and Tools, EOOLT 2011, Zurich, Switzerland, 2011.

[25] W. Schäfer and H. Wehrheim. Model-driven development with mechatronic uml. Graph transformations and model-driven engineering, pages 533$554,2010$.

[26] W. Schamai. Modelica modeling language (modelicaml) : A uml profile for modelica. Technical report, Linköping University, Department of Computer and Information Science, The Institute of Technology, 2009.

[27] C. Szyperski. Component Software: Beyond Object-Oriented Programming. Addison-Wesley, 1998. 